EUROPEAN ORGANIZATION FOR NUCLEAR RESEARCH

CERN-PPE/93-207

26 Nov 1993

\title{
A Study of Four-Fermion Processes at LEP
}

\author{
The L3 Collaboration
}

\begin{abstract}
We have studied the four-fermion processes $\mathrm{e}^{+} \mathrm{e}^{-} \rightarrow$ eeee, ee $\mu \mu$, ee $\tau \tau, \mu \mu \mu \mu$, $\mu \mu \tau \tau$, eeqq and $\mu \mu \mathrm{qq}$ with the L3 detector at LEP. For an integrated luminosity of 36 $\mathrm{pb}^{-1}$, corresponding to 960,000 hadronic $\mathrm{Z}$ decays, we find a total of 67 candidate events. The rate and kinematical distributions are found to be consistent with first order Monte Carlo calculations based on the Standard Model. No significant structure is seen in the dilepton invariant or recoil mass spectra.
\end{abstract}

(To be published in Physics Letters B) 


\section{Introduction}

In this paper we present a study of the four-fermion processes $\mathrm{e}^{+} \mathrm{e}^{-} \rightarrow$ eeee, ee $\mu \mu$, ee $\tau \tau$, $\mu \mu \mu \mu, \mu \mu \tau \tau$, eeqq and $\mu \mu \mathrm{qq}$ at $\sqrt{s} \simeq M_{\mathrm{Z}}$. Within the Standard Model (SM) there are many processes which can result in these four-fermion final states. At the $\mathrm{Z}$ resonance, with all final state particles being detected, the main contribution comes from the conversion of a virtual bremsstrahlung photon in the initial or final state (Fig. 1). Rates and kinematical distributions for such SM processes have been evaluated [1,2]. Four-fermion events have an important potential for discovery of any particle coupled to the Z. In L3 a pure sample can be obtained and the good energy/momentum resolution for leptons can be used to search for new particles coming from processes such as $\mathrm{e}^{+} \mathrm{e}^{-} \rightarrow \mathrm{Z} \rightarrow \mathrm{XZ}^{*} \rightarrow \quad \ell \ell \bar{f}$, where $\mathrm{X}$ or $\mathrm{Z}^{*}$ decay into an $\ell \ell$ or $f \bar{f}$ pair. In addition, it is important to check the SM predictions because these channels form an important background for Higgs searches [3,4].

The above channels have been studied earlier at LEP [5]. An L3 search for the $\mathrm{e}^{+} \mathrm{e}^{-} \rightarrow \tau \tau \tau \tau$ and $\tau \tau$ qq channels is described elsewhere [4].

For this analysis we used $36 \mathrm{pb}^{-1}$ of data taken around the $\mathrm{Z}$ peak, at center of mass energies between 88.5 and $93.7 \mathrm{GeV}$, during the LEP 1991 and 1992 runs. The data correspond to 960,000 hadronic $\mathrm{Z}$ decays.

\section{The L3 Detector}

The L3 detector is designed to measure electrons, photons, muons and jets produced in $\mathrm{e}^{+} \mathrm{e}^{-}$ reactions with good spatial and energy resolution. Starting from the interaction point, the L3 detector is composed of the following subdetectors: a time expansion chamber (TEC) for tracking charged particles $\left(13^{\circ}<\theta<167^{\circ}\right)$; an electromagnetic calorimeter (ECAL) composed of Bismuth Germanium Oxide crystals, consisting of a barrel $\left(42^{\circ}<\theta<138^{\circ}\right)$ and endcaps $\left(10^{\circ}<\theta<37^{\circ}\right.$ and $\left.143^{\circ}<\theta<170^{\circ}\right)$; a hadron calorimeter (HCAL) with uranium absorber and proportional wire chambers $\left(5^{\circ}<\theta<175^{\circ}\right)$; a muon spectrometer (MUCH) consisting of multi-wire drift chambers $\left(35.8^{\circ}<\theta<144.2^{\circ}\right)$. These detectors are installed in a $12 \mathrm{~m}$ inner diameter solenoidal magnet which provides a uniform magnetic field of $0.5 \mathrm{~T}$ along the beam direction. A detailed description of the detector and its performance is given in ref. [6].

\section{Event Selection}

Below we present our selection of the four-lepton channels followed by channels with quark pairs.

\section{Four-Lepton Events}

\section{Preselection}

A preselection common to all four-lepton channels is applied. We require:

- less than 20 calorimetric clusters (rejecting qq events);

- for the total event energy, $E_{t o t}: E_{t o t} / \sqrt{s}>0.35$ (rejecting beam-gas events and twophoton events with large missing energy);

- for the number of TEC tracks, $N_{T E C}: 4 \leq N_{T E C} \leq 8$ (rejecting Bhabha, dimuon and qq events). 
An important residual background is $\mathrm{e}^{+} \mathrm{e}^{-} \rightarrow \tau^{+} \tau^{-}$events, in particular $\tau^{+} \tau^{-}$events with $1+3$ and $3+3$ prong decay modes. To reduce this background, we use the fact that, due to the boost of a highly energetic $\tau$, the angles between its decay products will be small. Examining the azimuthal angles of the tracks, $\phi$, we cut on the angle spanned by a group of tracks, $\Delta \phi$.

- We reject 4 -track events if $\Delta \phi<0.2 \mathrm{rad}$ for any group of 3 tracks. We reject 6 (or 5 ) track events if we can group these tracks into two groups of 3 tracks (or one group of 3 and one of 2) and both groups have $\Delta \phi<0.2 \mathrm{rad}$.

Background event samples are generated using BABAMC [7] for Bhabha events, KORALZ [8] for dimuon and $\tau^{+} \tau^{-}$events, and JETSET [9] for hadronic events. The response of the L3 detector is modeled with the GEANT3 [10] detector simulation program which includes the effects of energy loss, multiple scattering and showering in the detector materials and in the beam pipe. Hadronic showers in the calorimeters are simulated with the GHEISHA [11] program.

\section{Particle Identification}

We make an exclusive analysis. Because of the expected low rates these identification criteria are rather loose. We use the following categories: $\gamma, \mathrm{e}, \mu$ and jets.

Muons are detected and measured by the muon chambers and are required to point to the interaction point. Muons which have low energy or are outside the angular coverage of the muon spectrometer are identified using minimum ionizing particle (mip) tracks in the calorimeters associated with a TEC track having $p>1 \mathrm{GeV}$.

$\gamma$ or $\mathrm{e}^{ \pm}$candidates are identified by requiring an ECAL cluster with an energy larger than $50 \mathrm{MeV}$. We define $E_{\beta}^{E C A L}\left(E_{\beta}^{H C A L}\right)$ as the energy measured in ECAL (HCAL) in a cone of half-angle $\beta$ centered on the direction of the $e$ or $\gamma$ candidate; we require $E_{\beta=0.1 \text { rad }}^{H C A L}<0.05 E_{e}$, where $E_{e}$ is the measured energy of the particle assuming it is an $\mathrm{e}^{ \pm}$or $\gamma$. An $\mathrm{e}^{ \pm}$candidate is required to be associated with a TEC track.

A jet is defined as a cluster of particles having at least one TEC track, one ECAL cluster and $E_{\text {jet }}>1 \mathrm{GeV}$.

\section{Event Classification}

Using the above criteria, we require:

- for the eeee sample, four electrons;

- for the ee $\mu \mu$ sample, two electrons and two muons;

- for the $\mu \mu \mu \mu$ sample, four identified muons, of which at least two are identified in the muon chambers.

The total energy of the leptons (plus the energy of photons, if any) must exceed $80 \mathrm{GeV}$.

The classification of four-lepton events with a $\tau$ pair is more involved because of the complications of the different $\tau$ decay modes, and because of a remaining contamination by $\mathrm{e}^{+} \mathrm{e}^{-} \rightarrow \tau^{+} \tau^{-}$events. We therefore require electrons to be isolated from jets and mips by an angle $\alpha_{\mathrm{e}}>0.30 \mathrm{rad}$ and muons must be isolated from jets by an angle $\alpha_{\mu}>0.20 \mathrm{rad}$.

The SM four-lepton cross section increases with decreasing energy of the virtual photon. Four-lepton events therefore most often have two hard leptons and two soft ones. A $\tau^{+} \tau^{-}$pair is in general the pair coming directly from the $\mathrm{Z}$ and carries the larger part of the event energy. 
The selection criteria of the channels are:

- for the ee $\tau \tau$ sample:

(i) In channels where the $\tau$ 's decay exclusively into e's and $\mu$ 's, we select events with $4 \mathrm{e}$, $3 \mathrm{e}+1 \mu$, or $2 \mathrm{e}+2 \mu$ ( $\gamma$ 's may be present, but no hadronic jets). Letting $E_{\text {lept }}$ be the summed energy of these leptons and $E_{2 \tau}$ the visible energy of the two $\tau$ candidates (the two most energetic e's, the most energetic e and $\mu$, or the two $\mu$ 's for $4 \mathrm{e}, 3 \mathrm{e}+1 \mu$ and $2 \mathrm{e}+2 \mu$ events, respectively). We require:

- for $4 \mathrm{e} \quad: E_{\text {lept }}>0.7 E_{\text {tot }}, E_{2 \tau}>0.4 E_{\text {tot }}$ and $E_{\text {tot }}<80 \mathrm{GeV}$;

- for $3 \mathrm{e}+1 \mu: E_{l e p t}$ and $E_{2 \tau}$ cut as above;

- for $2 \mathrm{e}+2 \mu: E_{l e p t}, E_{2 \tau}$ and $E_{t o t}$ cut as above, and $E_{e}<E_{2 \mu}$.

For the events with three e's we further require that the cone spanned by these e's have an opening angle larger than $0.3 \mathrm{rad}$; this rejects the $\tau^{+} \tau^{-}$background where the pions of a 3-prong decay are largely absorbed by the BGO and feign e's.

(ii) In the channels with three isolated leptons and at least one jet $\left(E_{\text {jet }}>3 \mathrm{GeV}\right)$, we require $3 \mathrm{e}+$ jet or $2 \mathbf{e}+1 \mu+$ jet to be present in the event. Assuming the jet and either the $\mu$ or the most energetic e (in the case of events with $3 \mathrm{e}$ 's) to come from $\tau$ decays, we further require the angle between the $\tau$ 's to be at least $1.2 \mathrm{rad}$ and $E_{\text {jet }+ \text { lept }}>0.5 E_{\text {tot }}$. For events with 3e's, these e's should, as above, span an angle of at least $0.3 \mathrm{rad}$.

- for the $\mu \mu \tau \tau$ sample: The selection of this channel is analogous to the one for $\mathrm{e}^{+} \mathrm{e}^{-} \rightarrow$ ee $\tau \tau$. At least one of the muons must be identified by the muon chambers. In addition, we include events with two isolated muons (both detected by the muon chambers) and two hadronic jets $\left(E_{j e t}>3 \mathrm{GeV}\right)$, if the $\tau$ 's are separated by at least $1.2 \mathrm{rad}$ and $E_{2 \tau}>0.4 E_{t o t}$.

To remove events with a photon which has converted in detector material or the beam-pipe, we require $M_{e e}>50 \mathrm{MeV}$, where $M_{e e}$ is the minimum $\mathrm{e}^{+} \mathrm{e}^{-}$invariant mass. In addition, we require for the distance, $R$, from the interaction point to the secondary $\mathrm{e}^{+} \mathrm{e}^{-}$vertex (in the plane perpendicular to the beam and for the $\mathrm{e}^{+} \mathrm{e}^{-}$pair with the smallest $\left.M_{e e}\right): R<20 \mathrm{~mm}$.

\section{eeqq Events}

We expect the dominant contributions to the eeqq final state to come from the process $\mathrm{e}^{+} \mathrm{e}^{-} \rightarrow$ $\mathrm{Z} \gamma^{*} \rightarrow$ eeqq, where the $\gamma^{*}$ is a bremsstrahlung photon. If the electron pair comes directly from the $\mathrm{Z}$ we expect at least one highly energetic, isolated e ('Bhabha-like' events); if the qq pair comes directly from the $\mathrm{Z}$ we expect a high multiplicity hadronic event with two moderately isolated e's ('2e-inclusive' events).

An important ingredient of the event selection is the electron identification. However, due to larger backgrounds, more severe selection criteria than in the four-lepton selection are used. For the electrons we require $E_{e}>3 \mathrm{GeV}$ and the lateral energy distribution of the ECAL cluster to be consistent with the shape of an electromagnetic shower as determined from test beam studies.

We select events with at least two electrons, $E_{\text {tot }}>50 \mathrm{GeV}$ (to reject $\tau^{+} \tau^{-}$background) and the number of TEC tracks $>4$ (to reject radiative Bhabha events).

We now select 'Bhabha-like' eeqq events by imposing the following additional cuts:

- For the most energetic electron: $E_{e}>0.35 \sqrt{s}$ and $E_{30^{\circ}}^{E C A L+H C A L}<0.1 E_{e}$; and for the second most energetic electron $E_{15^{\circ}}^{E C A L+H C A L}<0.5 E_{e}$ (rejecting the inclusive electron background). Here the energy of the electron itself is excluded from the cone energy. 
To select '2e-inclusive' events we use the following criteria:

- At least one electron must be isolated in a cone of $30^{\circ}$ half-angle and a second one should be isolated in a cone of $15^{\circ}$, according to the following criteria: no other tracks (in $\phi$ only); no HCAL clusters; at most one other ECAL cluster; $E_{30^{\circ} \text { or } 15^{\circ}}^{E C A L+H C}<0.1 E_{e}$ (where tracks and clusters from other electron candidates are excluded here).

To safely remove photon conversions in the detector we finally impose: $M_{\mathrm{ee}}>2 \mathrm{GeV}$. Fig. 2 shows an example of an eeqq event.

\section{$\mu \mu \mathrm{qq}$ Events}

The main background to four-fermion $\mathrm{e}^{+} \mathrm{e}^{-} \rightarrow \mu^{+} \mu^{-} \mathrm{q} \overline{\mathrm{q}}$ events are hadronic decays of the $\mathrm{Z}$ with two opposite-sign muons coming from semileptonic decays of hadrons. Further, fake muons can arise in hadronic events when a hadron punches through the hadron calorimeter.

To isolate $\mathrm{e}^{+} \mathrm{e}^{-} \rightarrow \mu^{+} \mu^{-} \mathrm{q} \overline{\mathrm{q}}$ events, we require two opposite-sign muon tracks in the spectrometer coming from the interaction point. To reduce punchthrough, the muons must have a momentum of at least $3 \mathrm{GeV}$.

In order to ensure the presence of hadronic particles and to reject $\mathrm{e}^{+} \mathrm{e}^{-} \rightarrow \mu^{+} \mu^{-} \ell \ell$ events we impose $N_{T E C}>4$. Furthermore, to reduce $\mathrm{Z} \rightarrow$ qq events, we require $N_{T E C}<15$ and the number of calorimetric clusters to be smaller than 40 (four-fermion events, especially with light quarks, tend to have a smaller hadronic activity with respect to $\mathrm{Z} \rightarrow \mathrm{qq}$ events).

As in the eeqq analysis the isolation of the muons with respect to hadronic jets is of crucial importance. To measure the isolation we use for each of the two muons the quantities:

- $\mathcal{D}_{\mu}=E_{j e t} / p_{\mu}-1$, where $E_{\text {jet }}$ is the energy of the jet containing the muon and $p_{\mu}$ is the muon momentum. We require: $\min \left(\mathcal{D}_{\mu 1}, \mathcal{D}_{\mu 2}\right)<0.4$.

- $\mathcal{E}_{\mu}=E_{15^{\circ}}^{E C A L}-E_{3^{\circ}}^{E C A L}$. We require: $\min \left(\mathcal{E}_{\mu 1}, \mathcal{E}_{\mu 2}\right)<700 \mathrm{MeV}$.

\section{Monte Carlo Modeling}

To calculate the efficiencies and backgrounds for our selections, and to compare our data with the SM predictions, we use the FERMISV MC generator [2]. This generator includes all lowest order diagrams involving $\mathrm{Z}$ and photon propagators, thus including t-channel and two-photon physics (multiperipheral) contributions. Interference effects are also included. Initial and final state radiation are included in a leading-log approximation. Electroweak radiative corrections are calculated using the improved Born approximation. It should be noted, however, that QCD contributions are not included.

\section{Four-Lepton $M C$}

The cuts applied at the generator level to ensure efficient and reliable performance of the generator are: $15^{\circ}<\theta_{e}<165^{\circ}$ and $M_{e e}>50 \mathrm{MeV}$, where $M_{e e}$ is the $\mathrm{e}^{+} \mathrm{e}^{-}$invariant mass; $15^{\circ}<\theta_{\mu}<165^{\circ}$ and $E_{\mu}>1 \mathrm{GeV} ; 10^{\circ}<\theta_{\tau}<170^{\circ}$. The muon energy cut removes very soft muons which are absorbed by the calorimeters too quickly to be accurately identified. With these cuts, the following effective cross sections (at the $\mathrm{Z}$ peak) are obtained 1.9, 1.9, 1.2, 0.5 and $0.5 \mathrm{pb}$ for eeee, ee $\mu \mu$, ee $\tau \tau, \mu \mu \mu \mu$ and $\mu \mu \tau \tau$, respectively. The multiperipheral contributions to these cross sections are typically a few percent. 


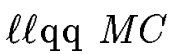

Events for the channels eeqq, $q=u, d, s, c$ and $b$ were generated separately. Generator level cuts are: $M_{e e}>2 \mathrm{GeV} ; M_{q q}>1 \mathrm{GeV}$; and $10^{\circ}<\theta_{e, q}<170^{\circ}$. The cut on the qq invariant mass excludes kinematic regions where QCD corrections, which are not taken into account by FERMISV (and which lead to processes like $\mathrm{e}^{+} \mathrm{e}^{-} \rightarrow \ell \ell V$, discussed below) are important. We find the following effective cross sections: $0.76,0.20,0.20,0.43$ and $0.07 \mathrm{pb}$ for $\mathrm{q}=\mathrm{u}, \mathrm{d}, \mathrm{s}, \mathrm{c}$ and $\mathrm{b}$, respectively.

For the generation of $\mu \mu \mathrm{qq}$ events we apply the following cuts on the generator level: $M_{\mu \mu}>$ $1 \mathrm{GeV} ; M_{q q}>1 \mathrm{GeV} ; 40^{\circ}<\theta_{\mu}<140^{\circ} ;\left|\vec{p}_{\mu}\right|>2 \mathrm{GeV}$. For the cross sections we find: 0.27 , $0.07,0.08,0.15$ and $0.03 \mathrm{pb}$ for $\mathrm{q}=\mathrm{u}, \mathrm{d}, \mathrm{s}, \mathrm{c}$ and $\mathrm{b}$, respectively.

\section{Vector Meson Dominance}

Vector Meson Dominance contributes to the process $\mathrm{e}^{+} \mathrm{e}^{-} \rightarrow \ell \ell q q$ via the reaction $\mathrm{e}^{+} \mathrm{e}^{-} \rightarrow$ $\ell \ell \rho[12]$, where the $\rho$ decays into $\pi^{+} \pi^{-1}$. An estimate of this cross section is required in order to have a more reliable $\ell$ qq cross section when $M_{q q} \leq 1 \mathrm{GeV}$. This process also contributes to the background of four-lepton final states when charged pions fake electrons. We use the FERMISV generator to create MC samples for these processes: we generate $\ell \ell \ell^{\prime} \ell^{\prime}\left(\ell^{\prime} \neq \ell\right)$ events (with $M_{\ell \ell}>50 \mathrm{GeV}$ and $10^{\circ}<\theta_{\ell, \ell^{\prime}}<170^{\circ}$ ) and replace the $\ell^{\prime} \ell^{\prime}$ system by a $\rho$ if $m_{\ell^{\prime} \ell^{\prime}} \approx m_{\rho}$ ( $m_{\rho}$ is drawn from the proper Breit-Wigner distribution). To estimate the cross section we again use the FERMISV program by scaling the event weights for $\ell l \ell^{\prime} \ell^{\prime}$ by $R=\sigma\left(\mathrm{e}^{+} \mathrm{e}^{-} \rightarrow\right.$ $\left.\pi^{+} \pi^{-}\right) / \sigma\left(\mathrm{e}^{+} \mathrm{e}^{-} \rightarrow \mu^{+} \mu^{-}\right)$near the $\rho$-resonance [13]. For each event, the value of $R$ is fixed by the mass of the virtual photon decaying into $\ell^{\prime} \ell^{\prime}$. Using the above cuts on $M_{\ell \ell}$ and $\theta_{\ell, \ell^{\prime}}$, we obtain the cross sections: $0.69,0.35$ and $0.31 \mathrm{pb}$ for $\mathrm{e}^{+} \mathrm{e}^{-} \rightarrow \mathrm{e}^{+} \mathrm{e}^{-} \rho, \mu^{+} \mu^{-} \rho$ and $\tau^{+} \tau^{-} \rho$, respectively. Including uncertainties in the calculational procedure and the MC statistics we assign a systematic error of $25 \%$ to the first number and $15 \%$ to the latter two. The value for $\sigma\left(\mathrm{e}^{+} \mathrm{e}^{-} \rightarrow \mu^{+} \mu^{-} \rho\right)$, after correction for initial state radiation effects, is in good agreement with the value from ref. [12].

\section{Results}

\section{Four-Lepton Events}

The number of observed events in each of the four-lepton channels are shown in Table 1 . Table 2 presents the selection efficiencies and misidentification probabilities determined by passing the MC events through the selection criteria. Table 1 also lists the MC predictions after convolution with the results in Table 2 . In the ee $\tau \tau$ channel we find $1.2 \pm 1.2$ background events from $\mathrm{Z} \rightarrow \mathrm{qq}$, and $3.0 \pm 1.2$ events from $\mathrm{Z} \rightarrow \tau \tau$. In total we observe 43 events compared to an expectation of $48.8 \pm 3.6$ events.

The observed numbers of events are stable against reasonable variations of the selection cuts. The corresponding efficiency variations result in a $5 \%$ uncertainty. We add another $11 \%$ error due to uncertainties in the MC modeling of time dependent detector inefficiencies and MC statistics. The error on the ee $\rho$ and $\mu \mu \rho$ background is dominated by the uncertainty in the cross section and the generation procedure for these processes. We conclude that the observed number of four-lepton events are in good agreement with their expected values.

\footnotetext{
${ }^{1}$ The processes $\mathrm{e}^{+} \mathrm{e}^{-} \rightarrow \ell \ell V$ for $V=\omega, \phi, J / \psi, .$. have negligible contributions due to their smaller electronic width and larger mass.
} 


\begin{tabular}{|l|c||c|c|c|}
\hline & \multicolumn{2}{|c|}{ Observed } & Expected & \multicolumn{2}{|c|}{ Backgrounds } \\
& Events & $4 \ell$ Events & $\tau \tau, \mathrm{qq}$ & $\ell \ell \rho$ \\
\hline \hline eeee & 14 & $15.8 \pm 2.0$ & - & $3.2 \pm \mathbf{0 . 8}$ \\
ee $\mu \mu$ & 12 & $10.1 \pm 1.4$ & - & $1.1 \pm \mathbf{0 . 2}$ \\
$\mu \mu \mu \mu$ & 3 & $3.4 \pm \mathbf{0 . 5}$ & - & - \\
ee $\tau \tau$ & 10 & $7.1 \pm 1.5$ & $4.2 \pm 1.7$ & $\mathbf{0} \pm_{0}^{0.1}$ \\
$\mu \mu \tau \tau$ & 4 & $3.9 \pm \mathbf{0 . 8}$ & $\mathbf{0} \pm_{0}^{1.3}$ & - \\
\hline total & 43 & $40.3 \pm 3.0$ & $4.2 \pm 1.7$ & $4.3 \pm \mathbf{0 . 8}$ \\
\hline
\end{tabular}

Table 1: The number of observed events in the individual \& lepton classes, the \& lepton $M C$ prediction with statistical and systematic errors combined, and the backgrounds for each event class.

\begin{tabular}{|l||ccccc|}
\hline \multicolumn{1}{|c||}{} & \multicolumn{5}{c|}{ Observed as: } \\
$(\%)$ & eeee & ee $\mu \mu$ & $\mu \mu \mu \mu$ & ee $\tau \tau$ & $\mu \mu \tau \tau$ \\
\hline \hline Process: & & & & & \\
eeee & 24.2 & 0.0 & 0.0 & 2.0 & 0.0 \\
ee $\mu \mu$ & 0.8 & 15.7 & 0.0 & 3.4 & 1.9 \\
$\mu \mu \mu \mu$ & 0.0 & 1.5 & 20.8 & 0.5 & 5.0 \\
ee $\tau \tau$ & 1.0 & 0.0 & 0.0 & 8.2 & 0.0 \\
$\mu \mu \tau \tau$ & 0.0 & 0.7 & 0.2 & 2.3 & 12.0 \\
\hline
\end{tabular}

Table 2: Efficiencies (diagonal elements) and misidentification probabilities (mixing between process (row) and observations (column)) in \%.

To quantify deviations from the SM we define $V$ to be the $\mathrm{e}^{+} \mathrm{e}^{-}$or $\mu^{+} \mu^{-}$pair with the lowest invariant mass. Results of regrouping the observed four-lepton events in $\ell \ell V$ classes are shown in Table 3. No excess of events is found in the four-lepton channels with a $\tau^{+} \tau^{-}$pair. The observed kinematical distributions also agree with those expected from SM processes. For example: Fig. $3 a$ shows the distribution of $\cos \alpha_{\ell V}$, where $\alpha_{\ell V}$ is the smallest angle between a lepton and the $V$; Fig. $3 b$ shows the acoplanarity distribution of the two most energetic leptons (for eeee, ee $\mu \mu$ and $\mu \mu \mu \mu$ only); Fig. 4 shows the distribution of the minimum and maximum dilepton invariant mass, $M^{\text {min }}\left(\ell^{+} \ell^{-}\right)$and $M^{\max }\left(\ell^{+} \ell^{-}\right)$, respectively. There is no significant clustering in any of the dilepton mass spectra. Fig. 4 a, however, shows several events with a rather high $M^{\text {min }}\left(\ell^{+} \ell^{-}\right)$; Table 4 compares the observed number of such events, as a function of a lower limit on $M^{\text {min }}\left(\ell^{+} \ell^{-}\right)$, to the number of expected events.

\begin{tabular}{|l|c|c|}
\hline$\ell \ell V$ & $\begin{array}{c}\text { Observed } \\
\text { events }\end{array}$ & $\begin{array}{c}\text { Expected } \\
\text { events }\end{array}$ \\
\hline \hline eeV & 20 & $22.6 \pm 2.6$ \\
$\mu \mu V$ & 10 & $12.3 \pm 1.9$ \\
$\tau \tau V$ & 13 & $13.9 \pm 2.5$ \\
\hline
\end{tabular}

Table 3: The result of regrouping the four-lepton events in $\ell \ell V$ categories (see text). 


\begin{tabular}{|c|c|c|}
\hline $\begin{array}{c}M^{\min }\left(\ell^{+} \ell^{-}\right) \\
(\mathrm{GeV}) \geq\end{array}$ & $\begin{array}{c}\text { Observed } \\
\text { events }\end{array}$ & $\begin{array}{c}\text { Expected } \\
\text { events }\end{array}$ \\
\hline \hline 3 & 5 & $4.5 \pm \mathbf{0 . 6}$ \\
4 & 5 & $3.6 \pm \mathbf{0 . 5}$ \\
15 & 2 & $\mathbf{0 . 8} \pm \mathbf{0 . 2}$ \\
20 & 1 & $\mathbf{0 . 4} \pm \mathbf{0 . 1}$ \\
\hline
\end{tabular}

Table 4: The number of observed events, as a function of the lower limit on the minimum dilepton mass in four-lepton events, $M^{\text {min }}\left(\ell^{+} \ell^{-}\right)$, compared to the SM expectation (see Fig. 4a).

\section{eeqq Events}

Combining the 'Bhabha-like' and the '2e-inclusive' event selection, we find 18 events. Using the $\mathrm{MC}$ samples for eeqq events, we find an overall eeqq efficiency of $29.9 \pm 2.5 \%$, where the error is due to MC statistics, to variations in the efficiency when varying the selection criteria within a reasonable range and to uncertainties in the MC modeling of detector inefficiencies. The expected number of eeqq events is then $15.0 \pm 1.2$. This value does not include QCD corrections. From MC studies we expect less than 1.3 background events from $\tau^{+} \tau^{-}$plus qq.

The observed number of eeqq events is in agreement with the SM expectation. Figs. 5 and 6 show the acollinearity angle and the invariant and recoil mass distribution of the electron pairs; the distributions are consistent with the expectations. No structures are observed in the mass distributions.

As a check, an independent analysis is performed for the 'Bhabha-like' part of the eeqq channel, the cuts on the cluster and track multiplicities are loosened (we require a minimum of 3 tracks instead of 5). Background is rejected by requiring that the clusters which are not associated with the two most energetic electrons, are isolated from the latter and not compatible with coming from electrons, $\mu$ 's, or converted $\gamma$ 's. We expect a total number of $13 \pm 3$ events. We select 17 events from the data ( 7 four-track events).

\section{$\mu \mu \mathrm{qq}$ Events}

With the selection criteria as described above, we find 6 events in our data. Using the fourfermion $\mathrm{e}^{+} \mathrm{e}^{-} \rightarrow \mu^{+} \mu^{-} \mathrm{q} \overline{\mathrm{q}} \mathrm{MC}$ we expect to observe $5.0 \pm 0.2$ events. MC studies show that backgrounds from qq, $\mu^{+} \mu^{-} \rho$ and other sources are negligible.

In Fig. 7 we show the $\mu^{+} \mu^{-}$invariant mass and the mass of the recoiling system. These distributions, and the total number of observed events, are in good agreement with the expectations.

\section{Conclusions}

We have studied the processes $\mathrm{e}^{+} \mathrm{e}^{-} \rightarrow$ eeee, ee $\mu \mu$, ee $\tau \tau, \mu \mu \mu \mu, \mu \mu \tau \tau$, eeqq and $\mu \mu \mathrm{qq}$ at LEP. We observed 43 events in the four-lepton channels and 24 events in the llqq channel. The number of observed events and their kinematical distributions are found to be consistent with Monte Carlo calculations based on the Standard Model. No significant structure is seen in the dilepton invariant or recoil mass spectra. 


\section{Acknowledgements}

We wish to express our gratitude to the CERN accelerator divisions for the excellent performance of the LEP machine. We acknowledge the efforts of all engineers and technicians who have participated in the construction and maintenance of this experiment.

\section{References}

[1] F.A. Berends, P.H. Daverveldt, and R. Kleiss, Comp. Phys. Comm. 40 (1986) 285;

R. Kleiss et al., in 'Z physics at LEP 1', CERN 89-08, Vol.3, (1989) p.98;

E.W.N. Glover, R. Kleiss and J.J. van der Bij, Z. Phys. C 47 (1990) 435.

[2] J. Hilgart, R. Kleiss and F. Le Diberder, Comp. Phys. Comm. 75 (1993) 191.

[3] O. Adriani et al., L3, Phys. Lett. B 303 (1993) 391.

[4] O. Adriani et al., L3, Z. Phys. C 57 (1993) 355.

[5] D. Decamp et al., ALEPH, Phys. Lett. B 263 (1991) 112;

P. Acton et al., OPAL, Phys. Lett. B 287 (1992) 389;

P. Abreu et al., DELPHI, Nucl. Phys. B 403 (1993) 3;

D. Buskulic et al., ALEPH, Phys. Lett. B 313 (1993) 299;

R. Akers et al., OPAL, CERN-PPE/93-145 (1993).

[6] B. Adeva et al., L3, Nucl. Instr. and Meth. A289 (1990) 35.

[7] M. Böhm, A. Denner and W. Hollik, Nucl. Phys. B 304 (1988) 687;

F.A. Berends, R. Kleiss and W. Hollik, Nucl. Phys. B 304 (1988) 712.

[8] S. Jadach and Z. Was, Comp. Phys. Comm. 36 (1985) 191;

See also, R. Kleiss et al., in 'Z Physics at LEP 1, CERN 89-08, Vol. 3, (1989) p.69.

[9] T. Sjöstrand, Comp. Phys. Comm. 39 (1986) 347;

T. Sjöstrand and M. Bengtsson, Comp. Phys. Comm. 43 (1987) 367.

[10] R. Brun et al., GEANT3 Users Guide, CERN/DD/EE/84.1.

[11] H. Fesefeldt, RWTH Aachen Report PITHA 85/02 (1985).

[12] L. Bergström and R.W. Robinett, Phys. Lett. B 245 (1990) 249;

K.J. Abraham and J.J. van der Bij, Phys. Lett. B 248 (1990) 199.

[13] We use the measured values as given in: L.M. Barkov et al., Nucl. Phys. B 256 (1985) 365. 


\section{The L3 Collaboration:}

A.Adam ${ }^{43}$ O.Adriani ${ }^{15}$ M.Aguilar-Benitez ${ }^{24}$ S.Ahlen, ${ }^{9}$ J.Alcaraz ${ }^{16}$ A.Aloisio, ${ }^{27}$ G.Alverson, ${ }^{10}$ M.G.Alviggi ${ }^{27}$ G.Ambrosi ${ }^{32}$ Q.An ${ }^{17}$ H.Anderhub ${ }^{46}$ A.L.Anderson ${ }^{14}$ V.P.Andreev ${ }^{36}$ T.Angelescu ${ }^{11}$ L.Antonov ${ }^{40}$ D.Antreasyan? P.Arce, ${ }^{24}$ A.Arefiev ${ }^{26}$ A.Atamanchuk, ${ }^{36}$ T.Azemoon, T.Aziz, P.V.K.S.Baba, ${ }^{17}$ P.Bagnaia ${ }^{35}$ J.A.Bakken, ${ }^{34}$ R.C.Ball ${ }^{3}$ S.Banerjee, K.Banicz ${ }^{43}$ J.Bao, R.Barillère, ${ }^{16}$ L.Barone, ${ }^{35}$ A.Baschirotto, ${ }^{25}$ R.Battiston, ${ }^{32}$ A.Bay, ${ }^{18}$ F.Becattini ${ }^{15}$ U.Becker ${ }^{14,46}$ F.Behner, ${ }^{46}$ J.Behrens, ${ }^{46}$ Gy.L.Bencze ${ }^{12}$ J.Berdugo ${ }^{24}$ P.Berges ${ }^{14}$ B.Bertucci ${ }^{32}$ B.L.Betev ${ }^{40,46}$ M.Biasini ${ }^{32}$ A.Biland, ${ }^{46}$ G.M.Bilei ${ }^{32}$ R.Bizzarri ${ }^{35}$ J.J.Blaising, G.J.Bobbink, ${ }^{16,2}$ R.Bock, A.Böhm ${ }^{1}$, B.Borgia ${ }^{35}$ M.Bosetti ${ }^{25}$ D.Bourilkov, ${ }^{29}$ M.Bourquin, ${ }^{18}$ D.Boutigny, ${ }^{16}$ B.Bouwens, E.Brambilla, ${ }^{27}$ J.G.Branson, ${ }^{37}$ I.C.Brock ${ }^{33}$ M.Brooks ${ }^{22}$ A.Bujak ${ }^{43}$ J.D.Burger, ${ }^{14}$ W.J.Burger ${ }^{18}$ J.Busenitz ${ }^{42}$ A.Buytenhuijss ${ }^{29}$ X.D.Cai ${ }^{17}$ M.Capell ${ }^{14}$ M.Caria, ${ }^{32}$ G.Carlino, ${ }^{27}$ A.M.Cartacci ${ }^{15}$ R.Castello, ${ }^{25}$ M.Cerrada ${ }^{24}$ F.Cesaroni ${ }^{35}$ Y.H.Chang, ${ }^{14}$ U.K.Chaturvedi ${ }^{17}$ M.Chemarin, ${ }^{23}$ A.Chen, ${ }^{48}$ C.Chen, G.Chen, G.M.Chen, H.F.Chen, ${ }^{6}{ }^{6}$ H.S.Chen, M.Chen, ${ }^{6}$ W.Y.Chen, ${ }^{48}$ G.Chiefari ${ }^{27}$ C.Y.Chien, M.T.Choi ${ }^{41}$ S.Chung, ${ }^{14}$ C.Civinini ${ }^{15}$ I.Clare, ${ }^{14}$ R.Clare, ${ }^{14}$ T.E.Coan, ${ }^{22}$ H.O.Cohn ${ }^{30}$ G.Coignet, N.Colino ${ }^{16}$ A.Contin, S.Costantini ${ }^{35}{ }^{4}$ F.Cotorobai ${ }^{11}$ X.T.Cui ${ }^{17}$ X.Y.Cui ${ }^{17}$ T.S.Dai ${ }^{14}$ R.D'Alessandro, ${ }^{15}$ R.de Asmundis, ${ }^{27}$ A.Degré, K.Deiters, ${ }^{44}$ E.Dénes, ${ }^{12}$ P.Denes, ${ }^{34}$ F.DeNotaristefani ${ }^{35}$ M.Dhina, ${ }^{46}$ D.DiBitonto, ${ }^{42}$ M.Diemoz ${ }^{35}$ H.R.Dimitrov ${ }^{40}$ C.Dionisi ${ }^{35}$ M.Dittmar, ${ }^{46}$ L.Djambazov ${ }^{46}$ M.T.Dova, ${ }^{17}{ }^{\natural}$ E.Drago, ${ }^{27}$ D.Duchesneau, P.Duinker, ${ }^{2}$ I.Duran, ${ }^{38}$ S.Easo, ${ }^{32}$ H.El Mamouni ${ }^{23}$ A.Engler, ${ }^{33}$ F.J.Eppling, ${ }^{14}$ F.C.Erné, P.Extermann, ${ }^{18}$ R.Fabbretti, ${ }^{44}$ M.Fabre, ${ }^{44}$ S.Falciano, ${ }^{35}$ S.J.Fan, ${ }^{39}$ O.Fackler, ${ }^{21}{ }^{1 . F a y}{ }^{23}$ M.Felcini ${ }^{16}{ }^{16}$ T.Ferguson ${ }^{33}$ D.Fernandez ${ }^{24}$ G.Fernandez ${ }^{24}$ F.Ferroni, ${ }^{35}$ H.Fesefeldt, E.Fiandrini ${ }^{32}$ J.H. Field ${ }^{18}$ F.Filthaut ${ }^{29}$ P.H.Fisher, G.Forconi, ${ }^{18}$ L.Fredj $^{18}{ }^{18}$ K.Freudenreich ${ }^{46}$ W.Friebel ${ }^{45}$ M.Fukushima ${ }^{14}$ M.Gailloud ${ }^{20}$ Yu.Galaktionov, ${ }^{26,14}$ E.Gallo, ${ }^{15}$ S.N.Ganguli, P.Garcia-Abia, ${ }^{24}$ D.Gele, ${ }^{23}$ S.Gentile, ${ }^{35}$ N.Gheordanescu, ${ }^{11}$ S.Giagu, ${ }^{35}$ S.Goldfarb, ${ }^{20}$ Z.F.Gong, ${ }^{19}$ E.Gonzalez ${ }^{24}$ A.Gougas, D.Goujon, ${ }^{18}$ G.Gratta, ${ }^{31}$ M.Gruenewald ${ }^{16}$ C.Gu ${ }^{17}$ M.Guanziroli ${ }^{17}$ J.K.Guo, ${ }^{39}$ V.K.Gupta ${ }^{34}$ A.Gurtu, H.R.Gustafson, L.J.Gutay, ${ }^{33}$ K.Hangarter, A.Hasan, ${ }^{17}$ D.Hauschildt, ${ }^{2}$ C.F.He, ${ }^{39}$ J.T.He, T.Hebbeker, ${ }^{16}$ M.Hebert ${ }^{37}$ A.Hervé ${ }^{16}$ K.Hilgers, ${ }^{16}$ H.Hofer ${ }^{46}$ H.Hoorani ${ }^{18}$ G.Hu, ${ }^{17}$ G.Q.Hu ${ }^{39}$ B.Ille ${ }^{23}$ M.M.Ilyas, ${ }^{17}$ V.Innocente, ${ }^{16}$ H.Janssen, B.N.Jin, ${ }^{6}$ L.W.Jones, I.Josa-Mutuberria, ${ }^{16}$ A.Kasser, ${ }^{20}$ R.A.Khan, ${ }^{17}$ Yu.Kamyshkov, ${ }^{30}$ P.Kapinos ${ }^{36,45}$ J.S.Kapustinsky ${ }^{22}$ Y.Karyotakis, ${ }^{16}$ M.Kaur ${ }^{17}$ S.Khokhar ${ }^{17}$ M.N.Kienzle-Focacci ${ }^{18}$ J.K.Kim ${ }^{41}$ S.C.Kim, ${ }^{41}$ Y.G.Kim,${ }^{41}$ W.W.Kinnison, ${ }^{22}$ A.Kirkby ${ }^{31}$ D.Kirkby ${ }^{31}$ S.Kirsch ${ }^{45}$ W.Kittel ${ }^{29}$ A.Klimentov ${ }^{14,26}$ A.C.König ${ }^{29}$ E.Koffeman, ${ }^{2}$ O.Kornadt, ${ }^{1}$ V.Koutsenko, ${ }^{1,26}$ A.Koulbardis ${ }^{36}$ R.W.Kraemer ${ }^{33}$ T.Kramer, ${ }^{14}$ V.R.Krastev ${ }^{40,32}$ W.Krenz ${ }^{1}$ A.Krivshich ${ }^{36}$ H.Kuijten ${ }^{29}$ K.S.Kumar ${ }^{13}$ A.Kunin ${ }^{14,26}$ G.Landi, ${ }^{15}$ D.Lanske, S.Lanzano, ${ }^{27}$ A.Lebedev, ${ }^{14}$ P.Lebrun ${ }^{23}$ P.Lecomte, ${ }^{46}$ P.Lecoq ${ }^{16}$ P.Le Coultre, ${ }^{46}$ D.M.Lee, ${ }^{22}$ J.S.Lee, ${ }^{41}$ K.Y.Lee, ${ }^{41}$ I.Leedom, ${ }^{10}$ C.Leggett, J.M.Le Goff, ${ }^{16}$ R.Leiste, ${ }^{45}$ M.Lenti ${ }^{15}$ E.Leonardi, ${ }^{35}$ C.Li, ${ }^{19}{ }^{17}$ H.T.Li, P.J.Li ${ }^{39}$ J.Y.Liao, ${ }^{39}$ W.T.Lin, ${ }^{48}$ Z.Y.Lin ${ }^{19}$ F.L.Linde, B.Lindemann, ${ }^{1}$ L.Lista ${ }^{27}{ }^{2}$ Y.Liu ${ }^{17}$ W.Lohmann ${ }^{45}$ E.Longo, ${ }^{35}$ W.Lu, ${ }^{31}$ Y.S.Lu, ${ }^{6}$ J.M.Lubbers ${ }^{16}$ K.Lübelsmeyer, C.Luci ${ }^{35}$ D.Luckey ${ }^{7,14}$ L.Ludovici ${ }^{35}{ }^{15}$ L.Luminari $^{35}$ W.Lustermann, ${ }^{45}$ J.M.Ma ${ }^{6}$ W.G.Ma, ${ }^{19}$ M.MacDermott ${ }^{46}$ L.Malgeri, ${ }^{35}$ R.Malik ${ }^{17}$ A.Malinin ${ }^{26}$ C.Maña, ${ }^{24}$ M.Maolinbay ${ }^{46}$ P.Marchesini, ${ }^{46}$ F.Marion, A.Marin, J.P.Martin, ${ }^{23}$ L.Martinez-Laso, ${ }^{24}$ F.Marzano, ${ }^{35}$ G.G.G.Massaro, K.Mazumdar, ${ }^{8}$ P.McBride, ${ }^{13}$ T.McMahon ${ }^{43}$ D.McNally, ${ }^{46}$ M.Merk, ${ }^{33}$ L.Merola ${ }^{27}$ M.Meschini ${ }^{15}$ W.J.Metzger, ${ }^{29}$ Y.Mi ${ }^{20}$ A.Mihul ${ }^{11}{ }^{\text {G.B.Mills }}{ }^{22}$ Y.Mir, ${ }^{17}$ G.Mirabelli, ${ }^{35}$ J.Mnich, M.Möller, B.Monteleoni, ${ }^{15}$ R.Morand, S.Morganti, ${ }^{15}$ N.E.Moulai, ${ }^{17}$ R.Mount, ${ }^{31}$ S.Müller, A.Nadtochy, ${ }^{36}$ E.Nagy ${ }^{12}$ M.Napolitano ${ }^{27}$ F.Nessi-Tedaldi ${ }^{46}$ H.Newman, ${ }^{31}$ C.Neyer,${ }^{46}$ M.A.Niaz ${ }^{17}$ A.Nippe, H.Nowak ${ }^{45}$ G.Organtini ${ }^{35}$ D.Pandoulas, ${ }^{1}$ S.Paoletti, ${ }^{35}$ P.Paolucci, ${ }^{27}$ G.Pascale, ${ }^{35}$ G.Passaleva, ${ }^{15,32}$ S.Patricelli ${ }^{27}$ T.Paul, M.Pauluzzi, ${ }^{32}$ C.Paus, F.Pauss, ${ }^{46}$ Y.J.Pei ${ }^{1}$, S.Pensotti ${ }^{25}$ D.Perret-Gallix, ${ }^{4}$ J.Perrier ${ }^{18}$ A.Pevsner, D.Piccolo, ${ }^{27}$ M.Pieri, ${ }^{16}$ P.A.Pirouée, ${ }^{34}$ F.Plasil ${ }^{30}$ V.Plyaskin ${ }^{26}$ M.Pohl ${ }^{46}$ V.Pojidaev ${ }^{26,15}$ H.Postema ${ }^{14}$ Z.D.Qi ${ }^{39}$ J.M.Qian, K.N.Qureshi, ${ }^{17}$ R.Raghavan, ${ }^{8}$ G.Rahal-Callot ${ }^{46}$ P.G.Rancoita, ${ }^{25}$ M.Rattaggi, ${ }^{25}$ G.Raven, P.Razis ${ }^{28}{ }^{2}$.Read ${ }^{30}$ D.Ren, ${ }^{46}$ Z.Ren ${ }^{17}$ M.Rescigno, ${ }^{35}$ S.Reucroft ${ }^{10}$ A.Ricker, ${ }^{10}$ S.Riemann, ${ }^{45}$ B.C.Riemers,${ }^{43}$ K.Riles, O.Rind, ${ }^{3}$ H.A.Rizvi ${ }^{17}{ }^{7}$ S.Ro ${ }^{41}{ }^{1}$ F.J.Rodriguez ${ }^{24}$ B.P.Roe, M.Röhner, S.Röhner, L.Romero ${ }^{24}{ }^{3}$ S.Rosier-Lees, R.Rosmalen, ${ }^{29}$ Ph.Rosselet ${ }^{20}$ W.van Rossum, ${ }^{2}$ S.Roth, ${ }^{1}$ A.Rubbia, ${ }^{14}$ J.A.Rubio, ${ }^{16}$ H.Rykaczewski, ${ }^{46}$ M.Sachwitz ${ }^{45}$ J.Salicio, ${ }^{16}$ J.M.Salicio ${ }^{24}$ G.S.Sanders ${ }^{22}$ A.Santocchia, ${ }^{32}$ M.S.Sarakinos ${ }^{14}$ G.Sartorelli, ${ }^{7}{ }^{17}$ M.Sassowsky, G.Sauvage, C.Schäfer, V.Schegelsky ${ }^{36}$ D.Schmitz, P.Schmitz, ${ }^{1}$, M.Schneegans, ${ }^{4}$ H.Schopper, ${ }^{47}$ D.J.Schotanus ${ }^{29}$ S.Shotkin, ${ }^{14}$ H.J.Schreiber, ${ }^{45}$ J.Shukla, ${ }^{33}$ R.Schulte, K.Schultze, J.Schwenke, G.Schwering, C.Sciacca, ${ }^{17}{ }^{1}$ I.Scott ${ }^{13}$ R.Sehgal ${ }^{17}$ P.G.Seiler, ${ }^{44}$ J.C.Sens ${ }^{16,2}$ L.Servoli ${ }^{32}$ I.Sheer ${ }^{37}$ D.Z.Shen, ${ }^{39}$ S.Shevchenko, ${ }^{31}$ X.R.Shi ${ }^{31}$ E.Shumilov ${ }^{26}$ V.Shoutko, ${ }^{26}$ D.Son, ${ }^{41}$ A.Sopczak, ${ }^{16}$ V.Soulimov, ${ }^{27}$ T.Spickermann, 1 P.Spillantini ${ }^{15}$ R.Starosta, ${ }^{1}$ M.Steuer, ${ }^{74}$ D.P.Stickland, ${ }^{34}$ F.Sticozzi ${ }^{14}$ H.Stone, ${ }^{34}$ K.Strauch ${ }^{13}$ K.Sudhakar, G.Sultanov ${ }^{17}{ }^{8}$ L.Z.Sun ${ }^{19,17}$ G.F.Susinno, ${ }^{18}$ H.Suter, ${ }^{46}$ J.D.Swain ${ }^{17}$ A.A.Syed ${ }^{29}$ X.W.Tang, ${ }^{6}$ L.Taylor ${ }^{10}$ G.Terzi ${ }^{25}$ Samuel C.C.Ting, ${ }^{14}$ S.M.Ting, ${ }^{14}$ O.Toker ${ }^{32}$ M.Tonutti, S.C.Tonwar, J.Tóth, ${ }^{12}$ A.Tsaregorodtsev, ${ }^{36}$ G.Tsipolitis, ${ }^{33}$ C.Tully ${ }^{34}$ K.L.Tung, ${ }^{6}$ J.Ulbricht, ${ }^{46}$ L.Urbán, ${ }^{12}$ U.Uwer, E.Valente, ${ }^{35}$ R.T.Van de Walle ${ }^{29}{ }^{1}$ I.Vetlitsky, ${ }^{26}$ G.Viertel ${ }^{46}{ }^{16}$ P.Vikas, ${ }^{17}$ U.Vikas, ${ }^{17}$ M.Vivargent, H.Vogel ${ }^{33}$ H.Vogt ${ }^{45}$ I.Vorobiev, ${ }^{13,26}$ A.A.Vorobyov ${ }^{36}$ L.Vuilleumier, ${ }^{20}$ M.Wadhwa, W.Wallraff, C.Wang, ${ }^{14}$ C.R.Wang, ${ }^{19}$ X.L.Wang, ${ }^{19}$ Y.F.Wang, ${ }^{14}$ Z.M.Wang ${ }^{17,19}$ A.Weber ${ }^{1}$, J.Weber $^{46}{ }^{16}$ R.Weill ${ }^{20}$ T.J.Wenaus, ${ }^{21}$ J.Wenninger ${ }^{18}$ M.White, ${ }^{14}$ C.Willmottt ${ }^{24}$ F.Wittgenstein ${ }^{16}$ D.Wright ${ }^{34}$ S.X.Wu, ${ }^{17}$ S.Wynhoff, B.Wysłouch, ${ }^{14}$ Y.Y.Xie, ${ }^{39}$ J.G.Xu, Z.Z.Xu, ${ }^{69}$ Z.L.Xue, ${ }^{39}$ D.S.Yan, ${ }^{39}$ B.Z.Yang, ${ }^{19}$ C.G.Yang, ${ }^{6}$ G.Yang, ${ }^{17}$ C.H.Ye, ${ }^{17}$ J.B.Ye, ${ }^{19}$ Q.Ye, ${ }^{17}$ S.C.Yeh, ${ }^{48}$ Z.W.Yin, ${ }^{39}$ J.M.You, ${ }^{17}$ N.Yunus, ${ }^{17}$ M.Yzerman, C.Zaccardelli, ${ }^{31}$ P.Zemp ${ }^{46}$ M.Zeng, ${ }^{17}$ Y.Zeng, D.H.Zhang, Z.P.Zhang, ${ }^{19,17}$ B.Zhou, G.J.Zhou, J.F.Zhou, R.Y.Zhu, ${ }^{31}$ A.Zichichi, ${ }^{76,17}$ B.C.C.van der Zwaan. 
1 I. Physikalisches Institut, RWTH, 52056 Aachen, FRG ${ }^{\S}$

III. Physikalisches Institut, RWTH, 52056 Aachen, FRG ${ }^{\S}$

2 National Institute for High Energy Physics, NIKHEF, NL-1009 DB Amsterdam, The Netherlands

3 University of Michigan, Ann Arbor, MI 48109, USA

4 Laboratoire d'Annecy-le-Vieux de Physique des Particules, LAPP,IN2P3-CNRS, BP 110, F-74941

Annecy-le-Vieux CEDEX, France

5 Johns Hopkins University, Baltimore, MD 21218, USA

6 Institute of High Energy Physics, IHEP, 100039 Beijing, China

7 INFN-Sezione di Bologna, I-40126 Bologna, Italy

8 Tata Institute of Fundamental Research, Bombay 400 005, India

9 Boston University, Boston, MA 02215, USA

10 Northeastern University, Boston, MA 02115, USA

11 Institute of Atomic Physics and University of Bucharest, R-76900 Bucharest, Romania

12 Central Research Institute for Physics of the Hungarian Academy of Sciences, H-1525 Budapest 114, Hungary ${ }^{\frac{1}{4}}$

13 Harvard University, Cambridge, MA 02139, USA

14 Massachusetts Institute of Technology, Cambridge, MA 02139, USA

15 INFN Sezione di Firenze and University of Florence, I-50125 Florence, Italy

16 European Laboratory for Particle Physics, CERN, CH-1211 Geneva 23, Switzerland

17 World Laboratory, FBLJA Project, CH-1211 Geneva 23, Switzerland

18 University of Geneva, CH-1211 Geneva 4, Switzerland

19 Chinese University of Science and Technology, USTC, Hefei, Anhui 230 029, China

20 University of Lausanne, $\mathrm{CH}-1015$ Lausanne, Switzerland

21 Lawrence Livermore National Laboratory, Livermore, CA 94550, USA

22 Los Alamos National Laboratory, Los Alamos, NM 87544, USA

23 Institut de Physique Nucléaire de Lyon, IN2P3-CNRS,Université Claude Bernard, F-69622 Villeurbanne Cedex, France

24 Centro de Investigaciones Energeticas, Medioambientales y Tecnologicas, CIEMAT, E-28040 Madrid, Spain

25 INFN-Sezione di Milano, I-20133 Milan, Italy

26 Institute of Theoretical and Experimental Physics, ITEP, Moscow, Russia

27 INF N-Sezione di Napoli and University of Naples, I-80125 Naples, Italy

28 Department of Natural Sciences, University of Cyprus, Nicosia, Cyprus

29 University of Nymegen and NIKHEF, NL-6525 ED Nymegen, The Netherlands

30 Oak Ridge National Laboratory, Oak Ridge, TN 37831, USA

31 California Institute of Technology, Pasadena, CA 91125, USA

32 INFN-Sezione di Perugia and Universitá Degli Studi di Perugia, I-06100 Perugia, Italy

33 Carnegie Mellon University, Pittsburgh, PA 15213, USA

34 Princeton University, Princeton, NJ 08544, USA

35 INFN-Sezione di Roma and University of Rome, "La Sapienza", I-00185 Rome, Italy

36 Nuclear Physics Institute, St. Petersburg, Russia

37 University of California, San Diego, CA 92093, USA

38 Dept. de Fisica de Particulas Elementales, Univ. de Santiago, E-15706 Santiago de Compostela, Spain

39 Shanghai Institute of Ceramics, SIC, Shanghai, China

40 Bulgarian Academy of Sciences, Institute of Mechatronics, BU-1113 Sofia, Bulgaria

41 Center for High Energy Physics, Korea Advanced Inst. of Sciences and Technology, 305-701 Taejon, Republic of Korea

42 University of Alabama, Tuscaloosa, AL 35486, USA

43 Purdue University, West Lafayette, IN 47907, USA

44 Paul Scherrer Institut, PSI, CH-5232 Villigen, Switzerland

45 DESY-Institut für Hochenergiephysik, 15738 Zeuthen, FRG

46 Eidgenössische Technische Hochschule, ETH Zürich, CH-8093 Zürich, Switzerland

47 University of Hamburg, 22761 Hamburg, FRG

48 High Energy Physics Group, Taiwan, China

$\S$ Supported by the German Bundesministerium für Forschung und Technologie

$\stackrel{+}{\ddagger}$ Supported by the Hungarian OTKA fund under contract number 2970 .

\# Also supported by CONICET and Universidad Nacional de La Plata, CC 67, 1900 La Plata, Argentina 


\section{Figure Captions}

- Figure 1: The dominant Feynman graphs for four-fermion processes at the $\mathrm{Z}$ resonance with all final state particles detected.

- Figure 2: An event display of the inner L3 detector elements, transverse to the beam axis. An eeqq candidate with an $\mathrm{e}^{+}$, an $\mathrm{e}^{-}$and two jets having energies of 14.2, 20.1, 23.5 and $29.3 \mathrm{GeV}$, respectively.

- Figure 3: (a) The cosine distribution of the angle between $\mathrm{V}$ (= lowest mass $\mathrm{e}^{+} \mathrm{e}^{-}$or $\mu^{+} \mu^{-}$pair) and the nearest other lepton. (b) The acoplanarity distribution of the two most energetic leptons (planar $\equiv \pi$ ).

- Figure 4: The minimum $\mathrm{e}^{+} \mathrm{e}^{-}$or $\mu^{+} \mu^{-}$invariant mass (a) and the maximum dilepton invariant mass (b) distributions in four-lepton events. Events with a high energetic $\tau \tau$ pair are excluded in the $M^{\max }(\ell \ell)$ distribution.

- Figure 5: The acollinearity distribution for electron pairs in eeqq events.

- Figure 6: The invariant mass (a) and recoil mass (b) distribution for the electron pair in eeqq events. The binning of plot (a) exceeds the ee-mass resolution.

- Figure 7: The invariant mass (a) and recoil mass (b) distribution for the muon pair in $\mu^{+} \mu^{-} \mathrm{q} \overline{\mathrm{q}}$ events. The binning of plot (a) exceeds the $\mu \mu$-mass resolution. 

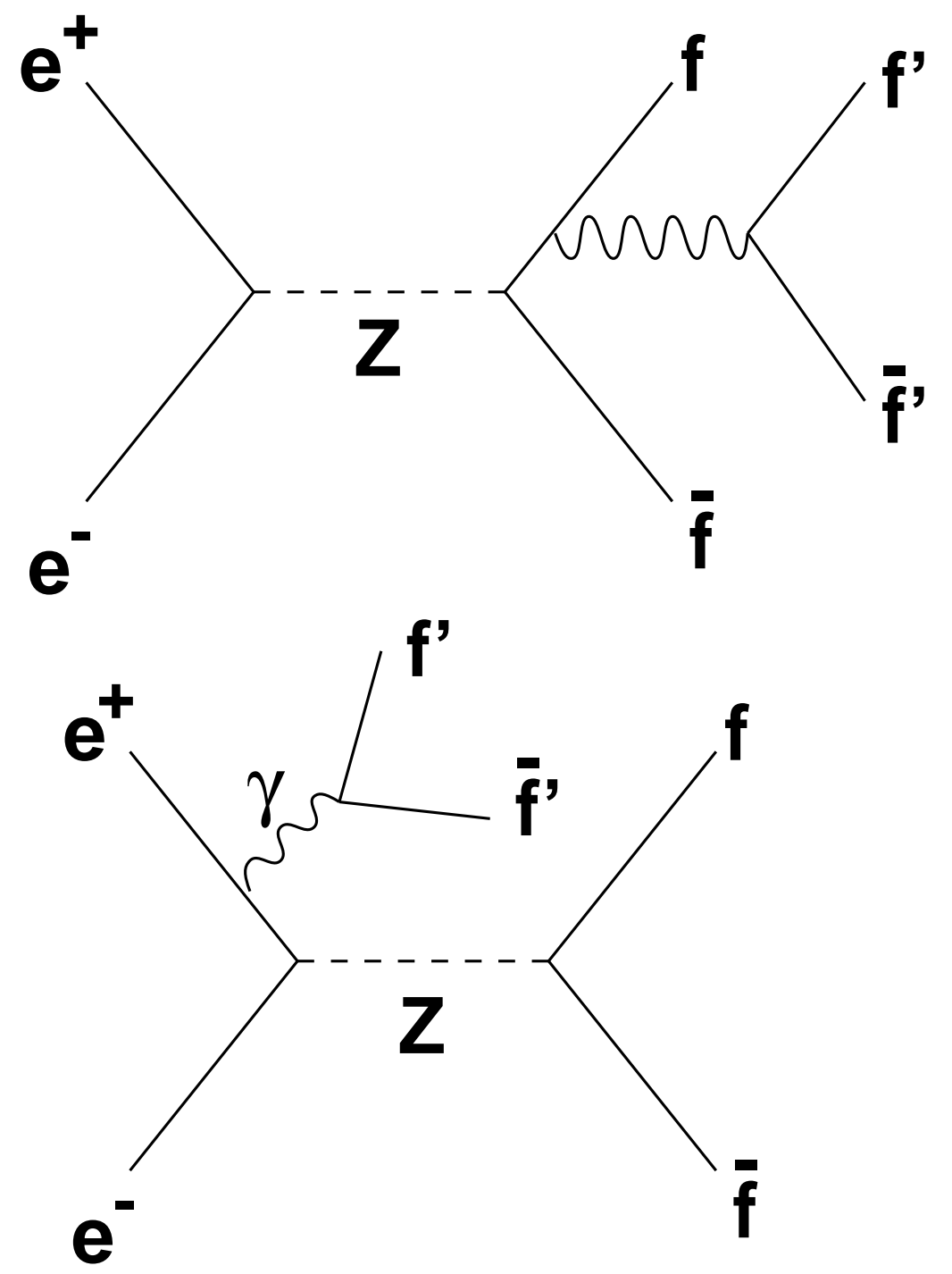

Figure 1 


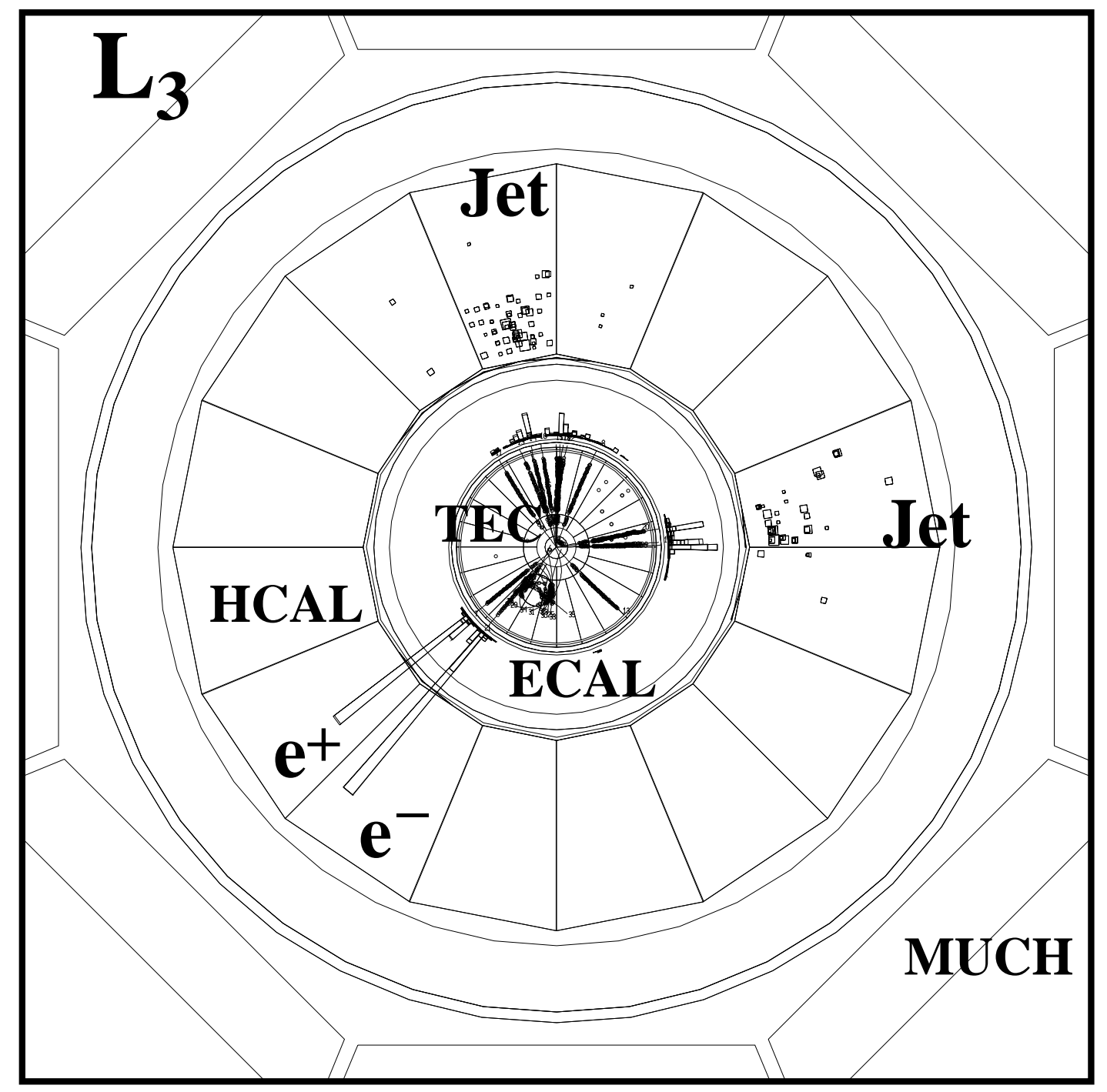

Figure 2 

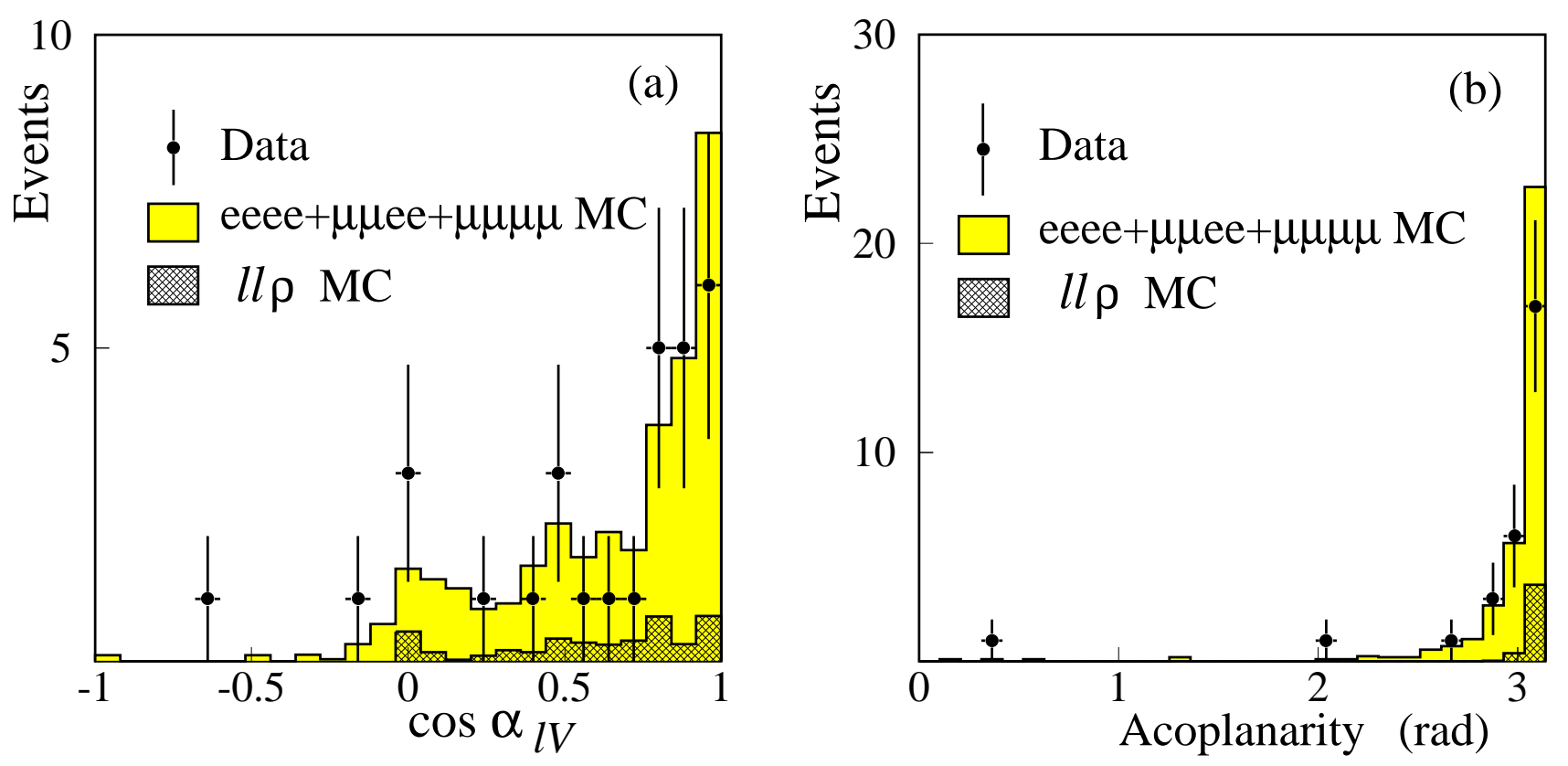

Figure 3 

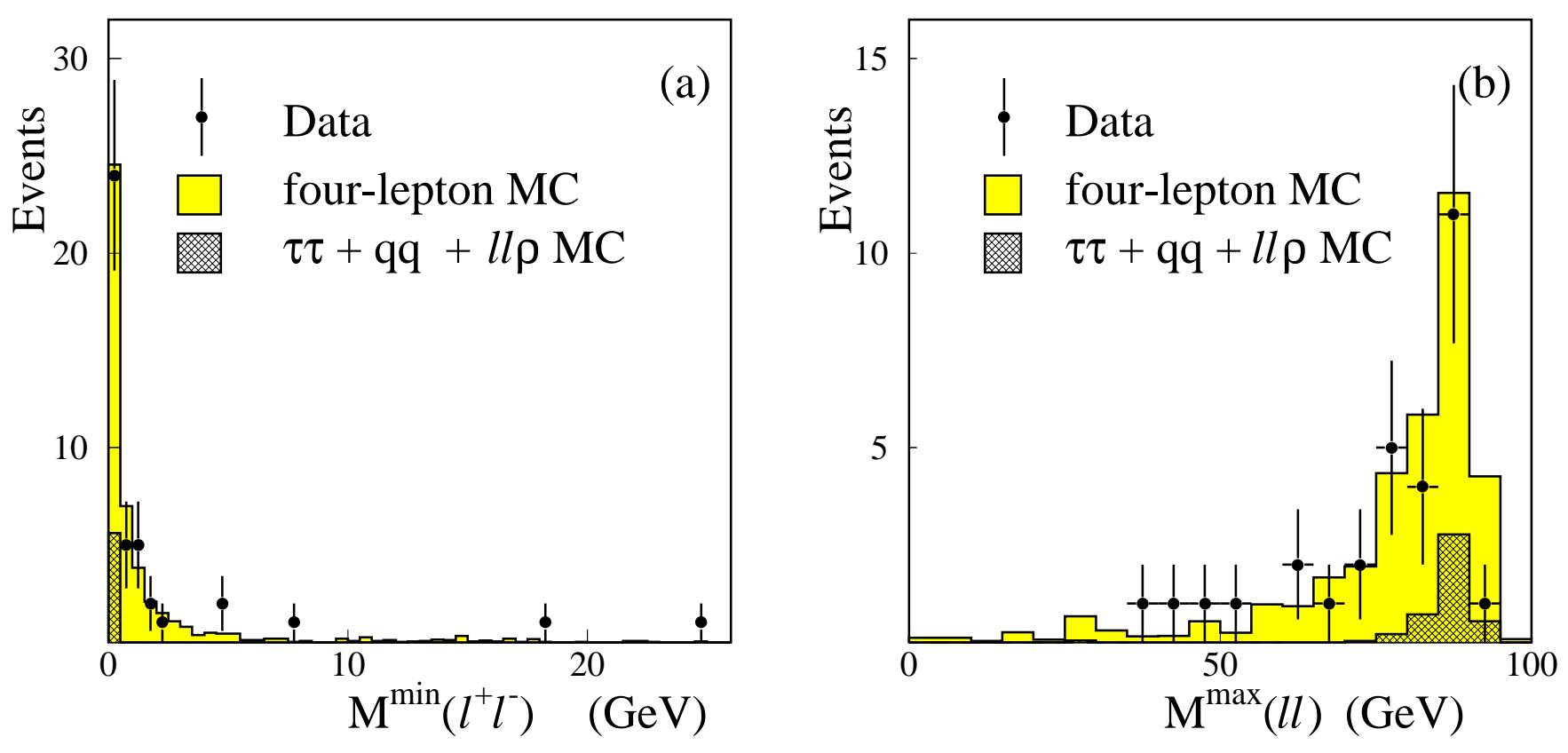

Figure 4 


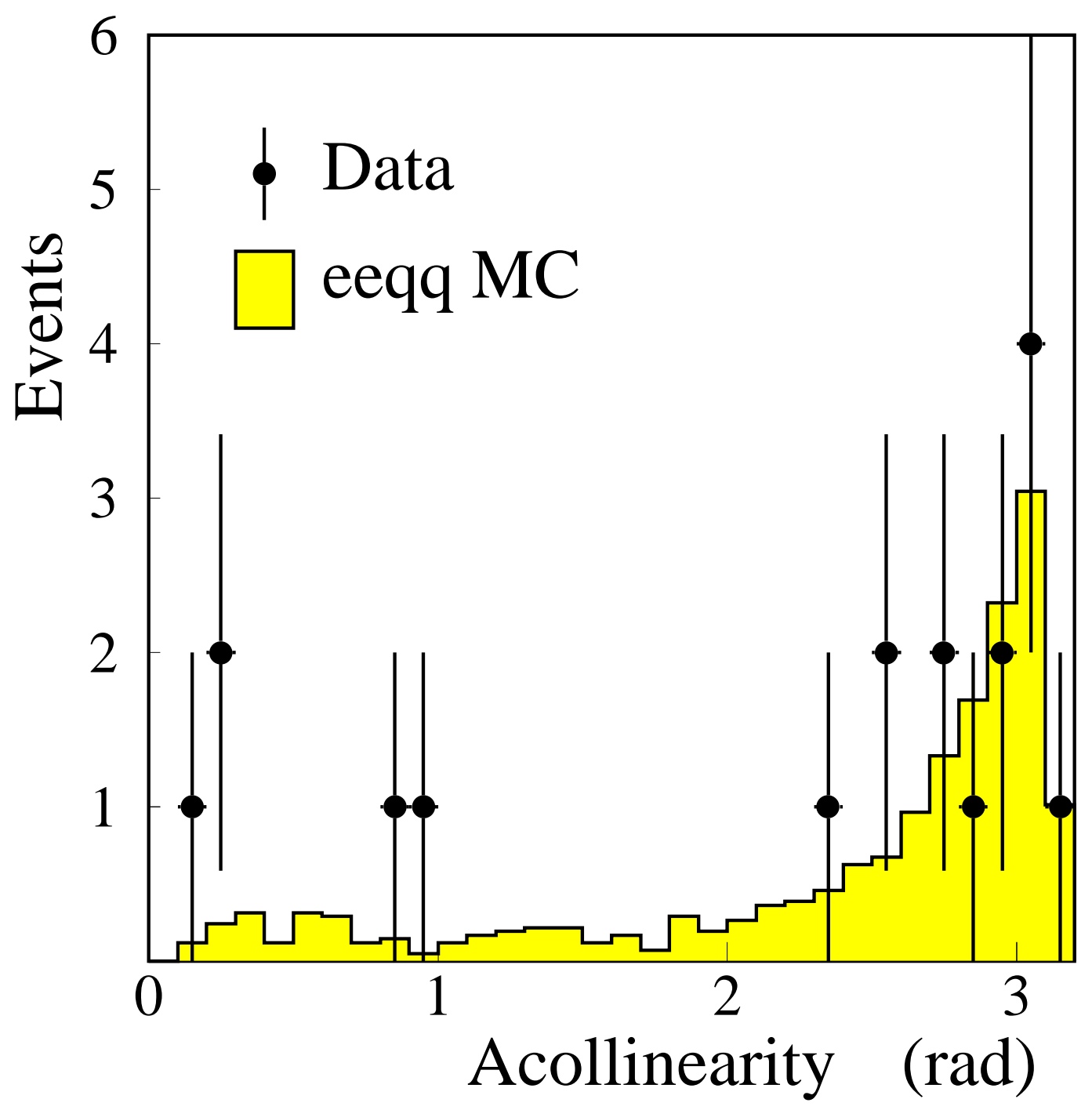

Figure 5 

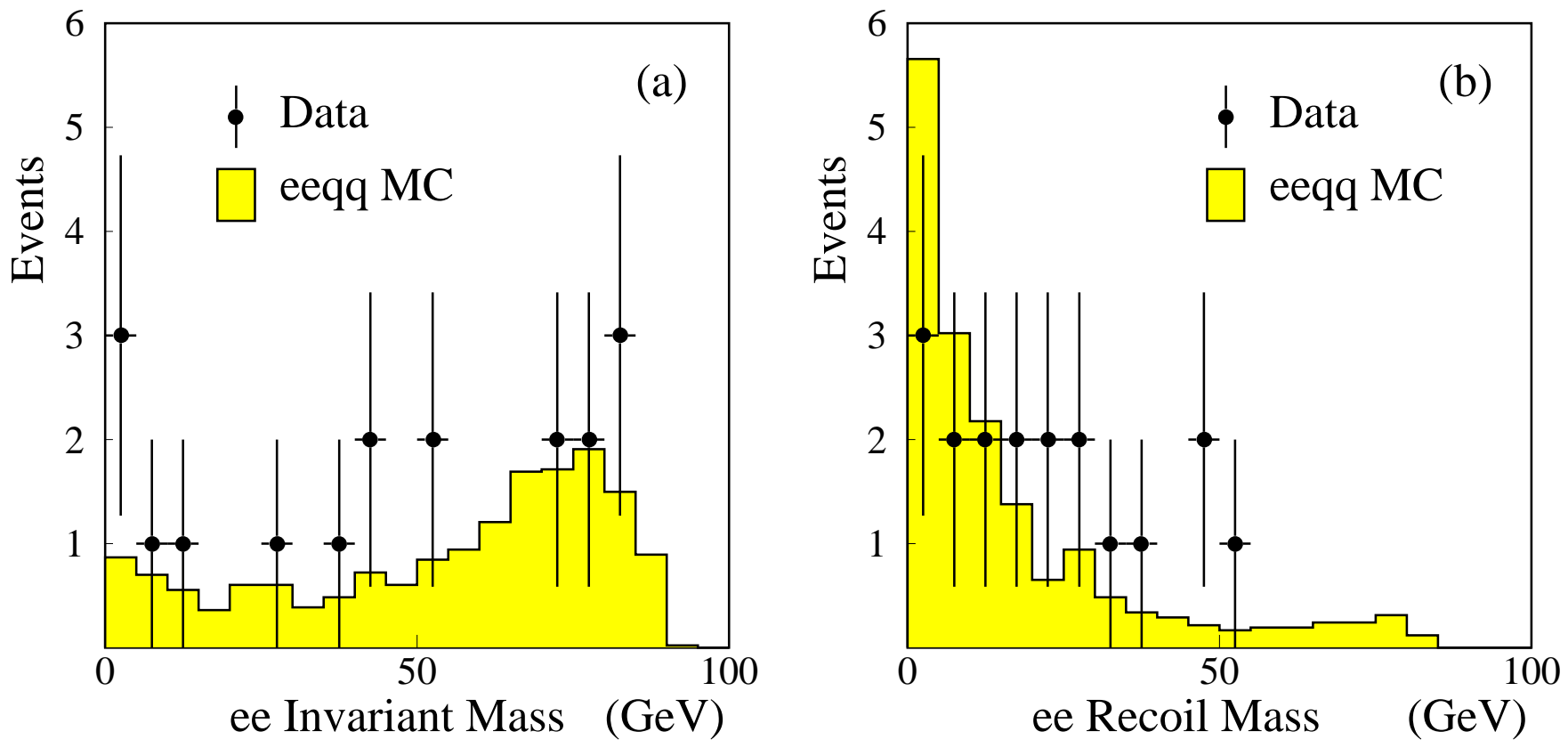

Figure 6 

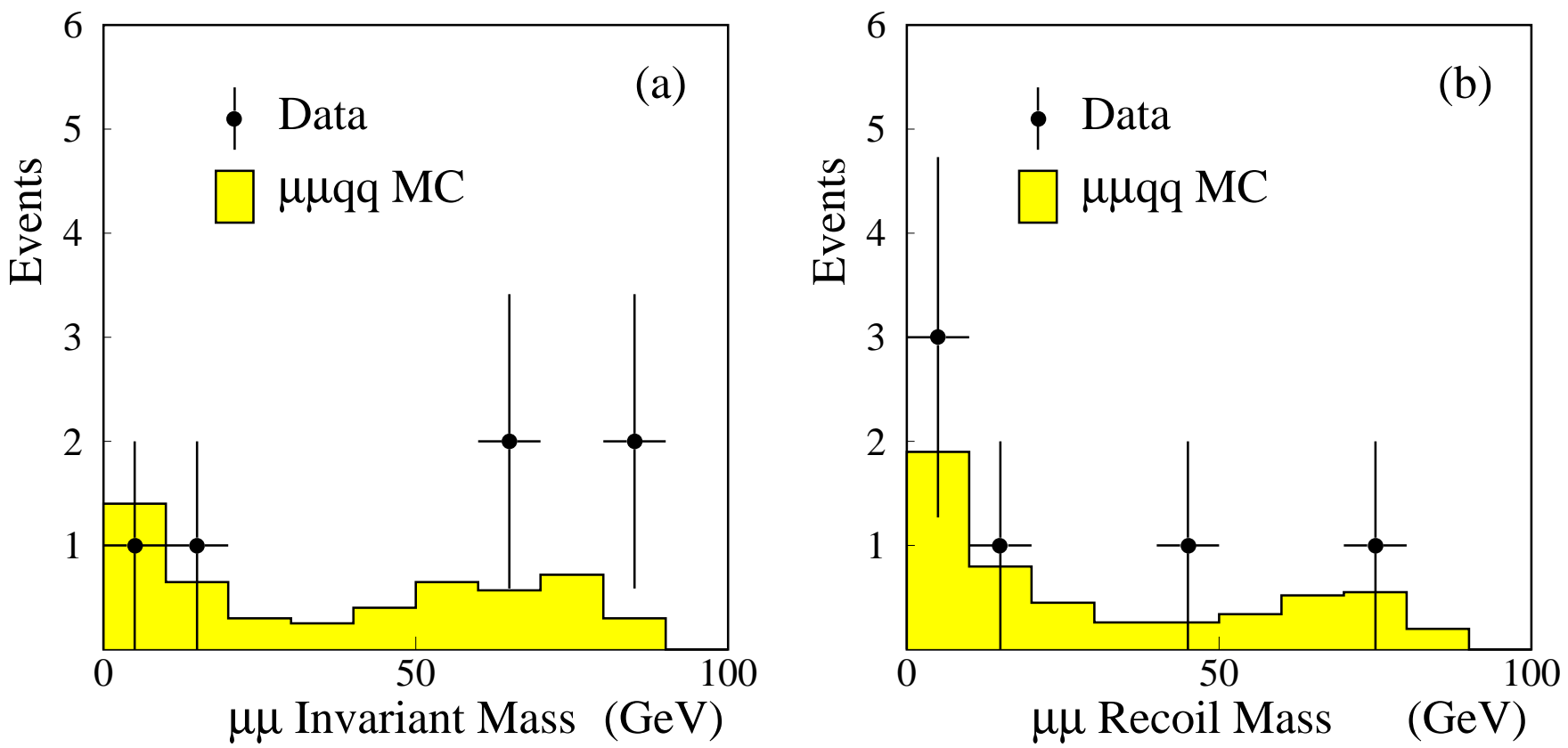

Figure 7 